\title{
Reading cells from within
}

A nanoscale field-effect transistor with a three-dimensional probe-presentation design can record intracellular potentials from single cells.

Nanotechnology devices have a vast range of applications, from electronics to energy production, and new developments are adding biology to this list. Nanowire and nanotube transistors can be used for ultrasensitive detection of biological markers and extracellular recording of membrane potential from cells. These tiny probes offer advantages over more conventional approaches such as electrodes: they are less invasive, make high-resolution measurements and have built-in capability for signal amplification. However, almost all examples of the prototypical nanoelectronic device, a nanoscale field-effect transistor (nanoFET), have been created on planar, twodimensional structures, making intracellular measurements difficult.

Charles Lieber's laboratory at Harvard University has been interested for years in developing interfaces between electric devices and biological systems. The group has now reported the development of a three-

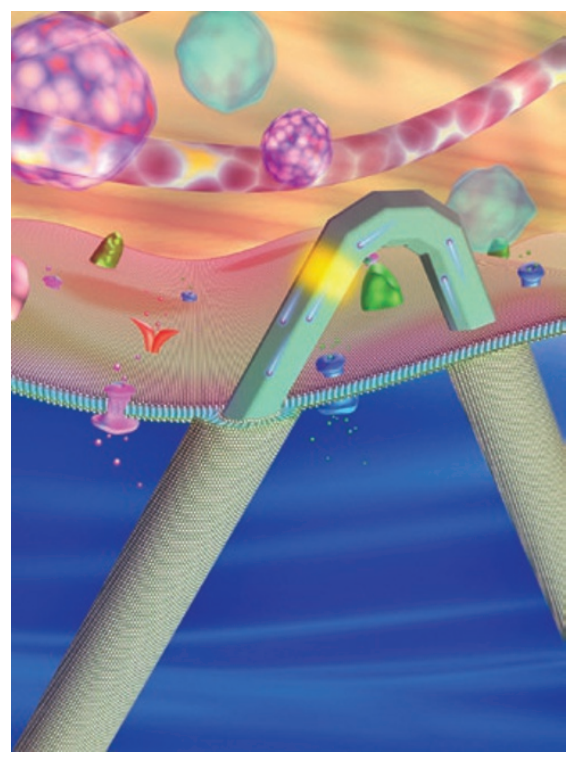

Schematic of a kinked-nanowire nanoFET sensor probing the intracellular region of a cell. The key nanoscale transistor element (yellow segment) is synthetically integrated at the tip of the acuteangle nanowire nanostructure. Image courtesy of Bozhi Tian and Charles Lieber.

dimensional, flexible nanoFET that can be used as a localized intracellular probe.
The researchers managed this by placing a nanoFET device at the tip of a bent silicon nanowire. Using a technique that allows reproducible kinks to be introduced into the nanowire, the group created a probe tip with an angle of 60 degrees. During synthesis, they incorporated the nanoscale FET at the tips of these 'bent nano-needles' and added the necessary source and drain electrical connections. Finally, the probe was lifted off the surface to facilitate its use for cellular measurements. The final outcome was a minute FET probe with pointlike morphology that allowed nanoscale interrogation of cells without interference from the bulky interconnects.

Lieber and colleagues coated the threedimensional nanoFET probes with phospholipid bilayers to facilitate minimally invasive cell entry and used them to record electrical signals from beating cardiomyocytes in culture. They made measurements by inverting the cell culture substrate and placing the cells, one by one, on top of a vertical nanoFET probe that was assembled on a chip. As well as performing highresolution extracellular recordings, the

\section{GENOMICS}

\section{EXPANDING HAPMAP}

\section{A haplotype map based on 11 global populations will provide a better understanding of human genome architecture.}

The human haplotype map (HapMap) has come a long way from its first public appearance in 2003. Phase 1 of the project saw a little over 1 million single-nucleotide polymorphisms (SNPs) genotyped in 270 individuals from four populations. The recently published HapMap 3 maps 1.6 million SNPs and around 800 copy-number polymorphisms in over 1,000 individuals from 11 populations as diverse as Japanese in Tokyo, Maasai in Kenya and Tuscans in Italy.

The HapMap project is a multinational effort to catalog genetic differences in humans. Initially the consortium's strategy was to use DNA microarrays containing common SNPs-polymorphisms with high minor allele frequencies-to genotype individuals and determine haplotyes, the blocks of SNPs that are always seen together in a population. These data provided a dense map of markers across the genome that proved useful, for example, in genome-wide association studies (GWAS) to establish connections between certain haplotypes and a phenotype of interest.
From its inception it was clear that HapMap would expand into different populations in future, but what became evident only over time was the importance of also including SNPs of lower frequency that current microarrays do not capture. "The current generation of [microarrays] is really quite a skewed version of the human genetic architecture," says Richard Gibbs of the Baylor College of Medicine, one of the HapMap project coordination leaders.

Hence, he and the other members of the HapMap 3 consortium decided on a two-pronged approach. They combined the use of microarrays to look at common SNPs in new populations with in-depth sequencing of ten 100-kilobase regions in 700 individuals to discover low-frequency, rare and private SNPs. These turned out to make up more than half of all variants in a population, with $99 \%$ of all new SNPs showing a minor allele frequency of less than $5 \%$.

The researchers were particularly intrigued by recurrent variants-SNPs that are rare, with a minor allele frequency of less than $0.5 \%$, but seen in geographically widely separated populations in the context of different haplotypes. These SNPs mark independently occurring events and will be of value for understanding the evolution of the human genome. 
probe was capable of penetrating cells and reading the electrical potential directly from within.

Additional work remains to develop this new synthetic nanoFET probe into a routine tool. The group is now working on designs that would allow researchers to manipulate it in a more traditional way, similarly to a patch-clamp micropipette. Gaining further control of the $z$-position of the probe and improving the optics used to guide the device will also greatly increase the utility of the system.

Work is underway to poke neurons with these nanoFET probes in acute brain slices, and the group hopes to use them soon in organisms in vivo. An exciting future application of this probe will be measuring membrane potentials directly from cellular organelles, something no other technology can currently do. As FETs can also be used for biochemical detection in cells, these probes could be adapted to simultaneously perform different types of intracellular measurements.

Lieber and his group are also interested in using the nanoFET probes as part of a scaffold to build biological tissue. "One dream is to merge arrays of these $3 \mathrm{D}$ nanoFETs with stem cells to drive and probe the differentiation, or with neural tissue for subsequent reimplantation so that one could have a seamless electrical interface in and out of the brain," he explains.

Lieber emphasizes that the most important next step will be to develop these nanoprobes in a format that makes them easily accessible to researchers. Now that the technology is here, biologists will surely be eager to peek into their favorite cells at the nanoscale.

\section{Erika Pastrana}

\section{RESEARCH PAPERS}

Tian, B. et al. Three-dimensional, flexible nanoscale field-effect transistors as localized bioprobes. Science 329, 830-834 (2010).

Surprisingly, the HapMap team also found that the rate of accumulation of rare variants in each individual did not flatten even after 700 people had been analyzed, supporting the idea that much larger sequencing efforts are called for to truly understand human genetic diversity.

Such sequencing efforts are underway in the 1000 Genomes Project, which aims to provide an extensive catalog of human genetic variation drawn from sequence data of thousands of people from 20 populations. Gibbs sees HapMap 3 as a forerunner for the 1000 Genomes Project, as it shows that deep sequencing uncovers variants that are hidden to array analysis; he quips that an appropriate summary of HapMap 3 could be, "Look what a good idea it is to sequence all these people."

Although HapMap 3 offers important insights into the variation in the human genome, a true data explosion will arise out of the 1000 Genomes project. These data will inform the design of new GWAS that may lead to a better understanding of the contribution of rare variants to complex traits.

\section{Nicole Rusk}

\section{RESEARCH PAPERS}

The International HapMap 3 Consortium. Integrating common and rare genetic variation in diverse human populations. Nature 467, 52-58 (2010).

\section{NEWS IN BRIEF}

\section{STEM CELLS}

\section{p53 knockout rats}

Transgenic technologies for rats have lagged behind those developed for mice. Tong et al. now describe the generation of $p 53$ (Tp53) knockout rats. They disrupted the p53 gene in germline-competent rat embryonic stem cells via homologous recombination and then introduced the cells into early-stage embryos to generate the knockout rat. This technology will permit the creation of a variety of gene-knockout rat strains for studying human disease.

Tong, C. et al. Nature 467, 211-213 (2010).

\section{PROTEOMICS}

\section{The aggregating proteome}

Protein aggregation occurs in neurodegenerative disorders such as Alzheimer's and Huntington's disease, but little is known about age-related protein aggregation in the absence of disease. David et al. performed a systematic proteomics analysis to address this question, using quantitative mass spectrometry to compare the insoluble fraction of proteins left after extracting all soluble proteins in young versus old roundworms. They found that hundreds of proteins become more insoluble in aging worms. David, D.C. et al. PLoS Biol. 8, e1000450 (2010).

\section{SIGNAL TRANSDUCTION}

\section{An integrated view of GPCR signaling}

$\mathrm{G}$ protein-coupled receptor (GPCR) signaling is typically measured by quantifying second messengers via fluorescence, but such assays do not reveal the integrated cellular response. Schröder et al. now demonstrate that a label-free, polarized light-based technology to monitor the dynamic mass redistribution (DMR) of molecules in the cell can be used to obtain a more complete view of GPCR signaling. This DMR assay allowed them to probe complex signaling patterns and map them to individual G-protein pathways.

Schröder, R. et al. Nat. Biotechnol. 28, 943-949 (2010).

\section{LAB ON A CHIP}

\section{Multiplexing in the billions}

In high-throughput suspension bioassays, a barcoding system is necessary for keeping track of different analytes. This can be done by attaching the analyte to a microparticle that is either spectroscopically encoded (by color) or graphically encoded (by pattern). Lee et al. now report a method combining spectroscopic and graphical encoding to generate potentially billions of unique magnetic microparticles carrying up to ten spatially separate colored barcodes, which can be decoded by ordinary microscopes. Lee, H. et al. Nat. Mater. 9, 745-749 (2010).

\section{BIOPHYSICS}

\section{Scanning ultrafast electron microscopy}

Scanning electron microscopy (SEM) can be used to obtain three-dimensional-like images of surfaces. Ultrafast electron microscopy allows imaging in both space and time at very high resolution. Yang et al. now combine these modalties to develop scanning ultrafast electron microscopy (SUEM), a four-dimensional imaging technique with nanometer and subpicosecond spatiotemporal resolution. They show that SUEM can be used to rapidly obtain three-dimensional pictures of biological specimens, including an ant's setae and an erythrocyte. Yang, D.-S. et al. Proc. Natl. Acad. Sci. USA 107, 14993-14998 (2010). 